\title{
An Efficient Outpatient Scheduling Approach
}

\author{
Haibin Zhu, Senior Member, IEEE, Ming Hou, Senior Member, IEEE, Chun Wang, Member, IEEE, \\ and MengChu Zhou, Fellow, IEEE
}

\begin{abstract}
Outpatient scheduling is considered as a complex problem. Efficient solutions to this problem are required by many health care facilities. Our previous work in Role-Based Collaboration (RBC) has shown that the group role assignment problems can be solved efficiently. Making connections between these two kinds of problems is meaningful. This paper proposes an efficient approach to outpatient scheduling by specifying a bidding method and converting it to a group role assignment problem. The proposed approach is validated by conducting simulations and experiments with randomly generated patient requests for available time slots. The major contribution of this paper is an efficient outpatient scheduling approach making automatic outpatient scheduling practical. The exciting result is due to the consideration of outpatient scheduling as a collaborative activity and the creation of a qualification matrix in order to apply the group role assignment algorithm.

Note to practitioners -As the "Age Wave" approaches, health care facilities are becoming relatively scarce worldwide compared with what are demanded. The varying availability, requirements, and preferences of both facilities and outpatients make the problem of scheduling outpatient appointments on health care facilities extremely challenging. Traditional manually operated scheduling systems based on phone calls are out of date although they are still widely used due to lack of new effective scheduling systems. To solve such a problem requires an efficient Web-based system to schedule the appointments instantly in order to make full use of those expensive and critical facilities. It is able to optimize concerned performance objectives in a clinical environment. The proposed approach provides a technical foundation for efficient Web-based scheduling systems, which can be applied directly to not only outpatient scheduling in the health care sector, but also in some other real-world scheduling applications.
\end{abstract}

Index Terms-Outpatient Scheduling, Roles, Agents, and Role Assignment

Manuscript received March 22, 2012, revised June 07, 2012. This work was supported in part by NSERC under Grant No. 262075, IBM Eclipse Innovation Grant, and DRDC - Toronto Grant.

H. Zhu is with the Department of Computer Science and Mathematics, Nipissing University, 100 College Drive, North Bay, Ontario, P1B 8L7, Canada (phone: 705-474-3450 ext. 4434; fax: 705-474-1947; e-mail: haibinz@nipissingu.ca).

M. Hou is with Defence Research \& Development Canada - Toronto, 1133 Sheppard Avenue West, Toronto, Ontario, M3M 3B9, Canada (phone: 416635-2063; fax: 416-635-2013; e-mail: Ming.Hou@ drdc-rddc.gc.ca).

C. Wang is with Concordia Institute for Information Systems Engineering, Concordia University, 1515 Ste Catherine West, EV 007.649, Montreal, H3G 1M8, Canada (phone: 514-8482424 ext. 5628; fax: 514-848-3171; email: cwang@ciise.concordia.ca).

M. C. Zhou is with the MoE Key Laboratory of Embedded System and Service Computing, Tongji University, Shanghai 200092, China (e-mail: mengchu@gmail.com).

\section{INTRODUCTION}

TN health care management, some facilities, such as magnetic resonance imaging (MRI) scanning or computed tomography (CT) scanning, are expensive and critical for certain disease diagnoses $[2,19]$. Normally expensive facilities must be pre-scheduled for unanticipated requirements of inpatients. Sometime later, pre-scheduled time slots may become available for outpatients to use. At the same time, outpatients may have different requests for the appointments and some appointments may not be available for some time slots, i.e., there are different constraints in assigning available time slots of an expensive device to available patients.

Therefore, instant re-scheduling is required at such a moment in terms of volume and urgency. Because the problem of efficient scheduling of patient appointments on expensive resources is complex and dynamic, it must be solved with an efficient system to re-schedule the appointments to avoid wastes and make full use of the expensive and critical facilities, i.e., the objective of outpatient scheduling is to find an appointment system for which a particular measure of performance is optimized in a clinical environment - an application of resource scheduling under uncertainty [2].

In Role-Based Collaboration (RBC) [23], Group Role Assignment (GRA) [27] is a complex problem for which the exhaustive-search based algorithm has exponential complexity. An efficient algorithm for GRA has been developed based on the Hungarian algorithm, also called Kuhn-Munkres (K-M) algorithm [11, 14]. It is of polynomial complexity. This work builds a system that transfers outpatient scheduling into a GRA problem.

\section{RELATED WORK}

It is well accepted that scheduling problems in health care services are important and complex. Much research is conducted in the fields of operational research and industrial engineering [2, 5]. However, very few attempts have been made to solve them through the development of practical systems. Cayirli and Veral [2] present general problem formulation and modeling considerations for effective scheduling systems, and provide taxonomy of methodologies used in previous literature. Godin and Wang [5] propose to allocate available diagnostic services timeslots to outpatients through an iterative bidding procedure which is a trigger to the idea of Call-For-Collaboration (CFC) in this paper. Gul et al. [6] compare several heuristics for scheduling an outpatient procedure center with respect to the competing criteria of 
expected patient waiting time and overtime. Guo et al. [7] present a simulation framework for the evaluation and optimization of scheduling rules. Gupta and Denton [8] state that many factors affect the performance of such systems including arrival and service time variability, patient and provider preferences, available information technology and the experience level of the scheduling staff. Kaandorp and Koole [10] model the outpatient appointment scheduling problem, and present a method to obtain optimal outpatient schedules in case of a finite number of possible arrivals. They do not mention the efficiency of their algorithms. Liu et al. [13] develop a framework and propose heuristic dynamic policies for scheduling patient appointments by considering that patients may cancel or not show up for their appointments. Their consideration is also another source of the problem presented in Section II, i.e., cancellation and no-show may release time slots for outpatients to reschedule. Patrick and Puterman [16] analyze the CT operations at Vancouver General Hospital, Canada and state that inpatient demands are fluctuating and the outpatient have priorities [2]. Our example is established based on this work [16]. Santibáñez et al. [19] develop a mixed integer programming model to schedule surgical blocks for each specialty into operating rooms and applied it to the hospitals in British Columbia Health Authority, considering operating room time availability and post-surgical resource constraints. Vermeulen et al. [20] present an adaptive approach to automatic optimization of resource calendars. The allocation of capacity to different patient groups is flexible and adaptive to the current and expected future situation.

\section{A REAL-World OUtPATIENT SCHEdULING PROBLEM}

The MRI lab of a hospital has a future 2-week schedule from Monday to Friday. Because some pre-occupied slots are released by in-patients, the newly available hours (slots) are presented as the shaded cells in Table I. Note that the preoccupied slots cannot be freed earlier than a period of time, e.g., two weeks, because the slots must be prepared for the inpatients. Hence, a two-week schedule is usually adopted.

Now, only one work day is left before the new schedule. The administration hopes to use as many unoccupied slots as possible. The question is how to assign the available slots to the most needed outpatients and instantly informs the outpatients the new scheduled time slots.

TABLE I. AN EXAMPLE OF A 2-WeEK SCHEDULE.

\begin{tabular}{|l|c|c|c|c|c|c|c|c|c|c|}
\hline & Mon & Tue & Wed & Thu & Fri & Mon & Tue & Wed & Thu & Fri \\
\hline $8: 00$ & 0 & 0 & 0 & 0 & 0 & 0 & 0 & 0 & 0 & 0 \\
\hline $9: 00$ & 1 & 1 & 1 & 1 & 1 & 1 & 1 & 1 & 1 & 1 \\
\hline $10: 00$ & 2 & 2 & 2 & 2 & 2 & 2 & 2 & 2 & 2 & 2 \\
\hline $11: 00$ & 3 & 3 & 3 & 3 & 3 & 3 & 3 & 3 & 3 & 3 \\
\hline $12: 00$ & 4 & 4 & 4 & 4 & 4 & 4 & 4 & 4 & 4 & 4 \\
\hline $13: 00$ & 5 & 5 & 5 & 5 & 5 & 5 & 5 & 5 & 5 & 5 \\
\hline $14: 00$ & 6 & 6 & 6 & 6 & 6 & 6 & 6 & 6 & 6 & 6 \\
\hline $15: 00$ & 7 & 7 & 7 & 7 & 7 & 7 & 7 & 7 & 7 & 7 \\
\hline $16: 00$ & 8 & 8 & 8 & 8 & 8 & 8 & 8 & 8 & 8 & 8 \\
\hline $17: 00$ & 9 & 9 & 9 & 9 & 9 & 9 & 9 & 9 & 9 & 9 \\
\hline $18: 00$ & 10 & 10 & 10 & 10 & 10 & 10 & 10 & 10 & 10 & 10 \\
\hline $19: 00$ & 11 & 11 & 11 & 11 & 11 & 11 & 11 & 11 & 11 & 11 \\
\hline
\end{tabular}

Conventionally, outpatient records in a database tell some outpatients' pre-filled requirements and availability in choosing adjacent time slots (also called requested bundles of time slots, or simply, requested bundles). Those outpatients who can come to fill newly available slots in Table I are shown in Table III. Based on such a table, an outpatient scheduling problem is modeled as an optimization problem and proved as an NP-hard problem [5]. To formalize the questions in this paper, we use the symbols in Table II.

\begin{tabular}{|c|c|}
\hline Symbol & Meaning \\
\hline A & A set of agents. \\
\hline$B$ & A bundle of time slots. \\
\hline$F^{t}(C, j)$ & The set formed by the elements of vector $C[j]$. \\
\hline $\mathcal{N}$ & The set of non-negative integers, i.e., $\{0,1,2, \ldots\}$. \\
\hline$P$ & $\begin{array}{l}\text { An } n \text {-dimensional vector of vectors of the bidding } \\
\text { blocks from outpatients. } P_{j} \text { is a vector with } k_{j}\left(1 \leq k_{j} \leq \mathrm{m}\right) \\
\text { bidding blocks requested by patient } j \in \Pi \text {. } P_{j} \text { also } \\
\text { expresses the preferences of patient } j \text { (if } l<\mathrm{k} \text { then } \\
\text { patient } j \text { prefers the } l^{\text {th }} \text { block } P_{j}[l] \text { to the } k^{\text {th }} \text { one } P_{j}[k] \text {, } \\
\left.0 \leq l, k<k_{j}, l \neq k\right) \text {. }\end{array}$ \\
\hline$P^{\prime}$ & $\begin{array}{l}\text { An } n \text {-dimensional vector of vectors of bidding sets (also } \\
\text { called bundles) of time slots from outpatients, where } P^{\prime}{ }_{j} \\
\text { is a vector with } k_{j}\left(1 \leq k_{j} \leq \mathrm{m} \text { ') bidding sets (bundles) of }\right. \\
\text { time slots requested by patient } j \in \Pi \text {. }\end{array}$ \\
\hline $\begin{array}{l}Q: \mathcal{A} \times R \rightarrow \\
{[0,1]}\end{array}$ & $\begin{array}{l}\text { A qualification matrix. } Q[i, r] \\
\text { qualification value of agent } I \text { for role } j \text {. }\end{array}$ \\
\hline$R$ & A set of roles. \\
\hline$S: \Omega \rightarrow \mathcal{N}$ & $S[i]$ expresses the size of block $I(I \in \Omega)$. \\
\hline$S^{\prime}: \Pi \rightarrow \mathcal{N}$ & $\begin{array}{l}S^{\prime}[j] \text { expresses the requested block size by patient } j(j \\
\in I \Pi) \text {. }\end{array}$ \\
\hline $\begin{array}{l}T: \mathcal{A} \times \mathbb{R \rightarrow} \\
\{0,1\}\end{array}$ & $\begin{array}{l}\text { An assignment matrix. } T[I, j]=1 \text { means that agent } I \text { is } \\
\text { assigned to role } j \text {, and } T[I, j]=0 \text { otherwise. }\end{array}$ \\
\hline$T^{\prime}$ & A vector of appointments for outpatients. \\
\hline$V: R \rightarrow A$ & $V[j]$ expresses the original agent assigned to role $j$. \\
\hline$W: R \rightarrow \mathcal{N}$ & $\begin{array}{l}\text { A vector to express the priority values of outpatients } \\
\text { (roles), where } \mathrm{W}_{j} \text { be the priority value assigned to } \\
\text { patient } j \in \Pi \text { or } j \in \mathbb{R} \text {. }\end{array}$ \\
\hline$g: \Omega \times \Pi \rightarrow[0,1]$ & $\begin{array}{l}\text { A preference scale to evaluate the relative preference } \\
\text { among different patients and requested blocks. }\end{array}$ \\
\hline $\begin{array}{l}H: \quad \Omega \times \Pi \rightarrow[0, \\
1]\end{array}$ & $\begin{array}{l}\text { The fitness of the requested size of the requested block } \\
\text { from } j \text { versus the available size of block } i \text {. }\end{array}$ \\
\hline$I$ & An element in $\Omega$ or $\mathcal{A}$. \\
\hline$J$ & An element in $\Pi$ or $\mathbb{R}$. \\
\hline$K$ & $\max \{m, n\}$ \\
\hline$m^{\prime}$ & The number of elements in $\Omega^{\prime}$, i.e., $\mid \Omega^{\prime}$. \\
\hline$m$ & The number of elements in $\Omega$ or $\mathcal{A}$, i.e., $|\Omega|$ or $|\mathcal{A}|$. \\
\hline$n$ & The number of elements in $\Pi$ or $\mathbb{R}$, i.e., $|\Pi|$ or $|\mathbb{R}|$. \\
\hline$w_{\max }$ & The maximum number in $W$. \\
\hline$X$ & $\begin{array}{l}\text { An assignment indicator. } X_{j}(B)=1 \text { if bundle } B \text { is } \\
\text { allocated to patient } j\left(B \subseteq \Omega^{\prime}, \quad 0 \leq j<\mathrm{n}\right), x_{j}(B)=0 \\
\text { otherwise. }\end{array}$ \\
\hline$\Delta$ & $\begin{array}{l}\text { The sum of the priority values of the scheduled } \\
\text { patients, i.e., } \sum_{B \in P^{\prime}} \sum_{j \in \Pi} x_{j}(B) \times W_{j} \text {. }\end{array}$ \\
\hline$\Delta_{1}$ & $\begin{array}{l}\text { The sum of the priority values of on the scheduled time } \\
\text { slots, i.e., } \sum_{i \in \Omega} \sum_{j \in \Pi} x_{j}(i) \times S^{\prime}[j] \times W_{j} \text {. }\end{array}$ \\
\hline$\Delta_{1}^{*}$ & $\max \{\Delta 1\}$ \\
\hline$\Pi$ & A set of the bidding outpatients. \\
\hline$\Omega$ & A set of available time slot blocks, or simply blocks. \\
\hline$\Omega^{\prime}, \Omega^{\prime \prime}$ & A set of available time slots. \\
\hline$\delta$ & $\min \{m, n\}$ \\
\hline$\zeta(P, i, j)$ & To express if agent $i$ belongs to $F^{t}(C, j)$. \\
\hline$\Psi$ & $\sum_{j \in \Pi} S^{\prime}[j] \times W_{j}$ \\
\hline
\end{tabular}

If each outpatient requires one bundle of time slots and we do not consider the preferences of patients among requested bundles, the problem can be formalized as [5]: 


$$
\max \Delta=\sum_{B \in P^{\prime}} \sum_{j \in \Pi} x_{j}(B) \times W_{j}
$$

subject to

$$
\begin{aligned}
& \forall j \in \Pi, \sum_{B \subseteq \Omega^{\prime}} x_{j}(B) \leq 1 \\
& \forall i \in \Omega^{\prime}, \sum_{B \ni i} \sum_{j \in \Pi} x_{j}(B) \leq 1 \\
& \forall j \in \Pi, \sum_{B \subseteq \Omega^{\prime}} x_{j}(B)=\sum_{B \in P_{j}^{\prime}} x_{j}(B) \\
& \forall B \subseteq \Omega^{\prime}, j \in \Pi x_{j}(B) \in\{0,1\}
\end{aligned}
$$

where constraint (2) ensures that each outpatient can be assigned at most one requested bundle of timeslots; (3) ensures that each time slot is assigned to only one patient; (4) ensures that the assigned bundle is requested by the patient; and (5) is a $0-1$ constraint. Note that $\sum_{B \ni i}$ in (3) is read as "sum for all $B$ that contains $i$.

TABLE III. THE AVAILABILITIES AND PREFERENCES OF OUTPATIENTS.

\begin{tabular}{|l|l|l|l|}
\hline ID & Name & $\begin{array}{l}\text { Priority } \\
\text { value }\end{array}$ & Outpatients' availabilities in a day \\
\hline P1 & Tom & 3 & $\begin{array}{l}\text { Monday: }\{7,8\}, \text { Wednesday: }\{2,3\}, \\
\text { Thursday: }\{3,4\}\end{array}$ \\
\hline P2 & Chris & 2 & Monday and Tuesday: $\{7,8,9\}$ \\
\hline P3 & Ana & 3 & Wednesday and Thursday: $\{3\}$ \\
\hline P4 & Bob & 2 & Wednesday: $\{1,2\}$, Thursday: $\{3,4\}$ \\
\hline P5 & Don & 1 & Any day: $\{0\}, \ldots,\{11\}$ \\
\hline P6 & Jane & 3 & Thursday: $\{7,8\}$ \\
\hline
\end{tabular}

TABle IV. AVAilable Time Slots (34).

\begin{tabular}{|l|c|c|c|c|c|c|c|c|c|c|}
\hline & Mon & Tue & Wed & Thu & Fri & Mon & Tue & Wed & Thu & Fri \\
\hline $8: 00$ & 0 & & & & & & & & & \\
\hline $9: 00$ & 1 & & 9 & & & & & 19 & & \\
\hline $10: 00$ & & 6 & 10 & & & & & 20 & & \\
\hline $11: 00$ & & 7 & 11 & & & & & 21 & 26 & \\
\hline $12: 00$ & & 8 & & & & & & & 27 & \\
\hline $13: 00$ & & & & & & & & 22 & & \\
\hline $14: 00$ & & & & 12 & & & 15 & 23 & 28 & \\
\hline $15: 00$ & 2 & & & 13 & & & 16 & 24 & 29 & \\
\hline $16: 00$ & 3 & & & 14 & & & 17 & 25 & 30 & \\
\hline $17: 00$ & 4 & & & & & & 18 & & 31 & \\
\hline $18: 00$ & 5 & & & & & & & & 32 & \\
\hline $19: 00$ & & & & & & & & & 33 & \\
\hline
\end{tabular}

To solve the problem described in (1)-(5), the available time

\begin{tabular}{|c|c|c|c|c|c|c|c|c|c|c|}
\hline $\begin{array}{l}\text { Patient } \\
\text { ID }\end{array}$ & \multicolumn{2}{|c|}{ Name } & \multicolumn{2}{|c|}{$\begin{array}{l}\text { Priority } \\
\text { value }\end{array}$} & \multicolumn{6}{|c|}{ Outpatients' requested bundles } \\
\hline $\mathrm{P} 1$ & \multicolumn{2}{|c|}{ Tom } & \multicolumn{2}{|l|}{3} & $\multicolumn{6}{|c|}{2,3},\{9,10\},\{19,20\},\{26,27\}$ \\
\hline $\mathrm{P} 2$ & \multicolumn{2}{|c|}{ Chris } & \multicolumn{2}{|l|}{2} & $\multicolumn{6}{|c|}{2,3,4},\{16,17,18\}$ \\
\hline P3 & \multicolumn{2}{|c|}{ Ana } & \multicolumn{2}{|l|}{3} & $\multicolumn{6}{|c|}{11},\{21\},\{26\}$ \\
\hline $\mathrm{P} 4$ & \multicolumn{2}{|c|}{ Bob } & \multicolumn{2}{|l|}{2} & $\multicolumn{6}{|c|}{9,10},\{19,20\},\{26,27\}$ \\
\hline P5 & \multicolumn{2}{|c|}{ Don } & \multicolumn{2}{|l|}{1} & $\multicolumn{6}{|c|}{0}, \ldots,\{33\}$ \\
\hline P6 & & Jane & \multicolumn{2}{|l|}{3} & $\multicolumn{6}{|c|}{13,14},\{29,30\}$ \\
\hline \multicolumn{11}{|c|}{ TABLE VI. ASSIGNED TIME SLOTS (NAMED SLOTS). } \\
\hline & Mon & Tue & Wed & Thu & Fri & Mon & Tue & Wed & The & Fri \\
\hline $8: 00$ & Don & & & & & & & & & \\
\hline 9:00 & 1 & & Bob & & & & & 19 & & \\
\hline $10: 00$ & & 6 & Bob & & & & & 20 & & \\
\hline $11: 00$ & & 7 & Ann & & & & & 21 & 26 & \\
\hline $12: 00$ & & 8 & & & & & & & 27 & \\
\hline $13: 00$ & & & & & & & & 22 & & \\
\hline $14: 00$ & & & & 12 & & & 15 & 23 & 28 & \\
\hline $15: 00$ & Tom & & & Jane & & & Chris & 24 & 29 & \\
\hline $16: 00$ & Tom & & & Jane & & & Chris & 25 & 30 & \\
\hline $17: 00$ & 4 & & & & & & Chris & & 31 & \\
\hline \begin{tabular}{|l|}
$18: 00$ \\
\end{tabular} & 5 & & & & & & & & 32 & \\
\hline \begin{tabular}{|l|}
$19: 00$ \\
\end{tabular} & & & & & & & & & 33 & \\
\hline
\end{tabular}
slots are numbered from 0-33 (Table IV), outpatients are numbered from $0-5$, and their availability is transferred to Table V.

TABle V. The aVAILABILITIES ANd PREFERENCES OF OUtPatients.

By using IBM ILOG CPLEX Optimization Studio V12.2 (ILOG), the problem is solved (Table VI) in $130 \mathrm{~ms}$ with the objective as 26 (Section $\mathrm{V}$ of the multimedia document). If the number of bundles and patients increases, the consumed time increases exponentially. Experiments also assert that such modeling could only work for relatively small size outpatient scheduling problems [5].

\section{COllaborative OUTPATIENT SCHEdULING - OUR STRATEGY}

In fact, outpatient scheduling is dynamic. Available time slots, the outpatients' availability and preference are changing. The information in Table III may not reflect the current states of all the outpatients. The model in (1)-(5) does not consider the preferences of outpatients.

In our strategy, the outpatient scheduling problem is considered as a collaborative action, i.e., the patients are collaborating on this scheduling work. The operation scenario is that, upon a change in the available time slots, the facility office or clinic sends out a Call for Collaboration (CFC) message by emails or calls to all the already registered (scheduled or not yet scheduled) outpatients, and some or all of them respond to the CFC message by bidding for bundles of time slots. The scheduling algorithm then makes optimal rescheduling based on their responses.

We assume that the time unit is in slot, and each outpatient has a priority value assigned by his/her doctor. The priority values of outpatients are taken as one indicator to express the ranking and competence on a time slot among outpatients. In this way, if a bundle of 2 time slots is assigned to a patient who has a priority value of 3 , we collect $3 \times 2$ into the sum of the priority values on the assigned time slots.

Then, the outpatient scheduling system is designed to find an assignment scheme for all patients such that the sum of priority values on the assigned slots is maximized and their preferences are satisfied.

We assume that the bidding patients may have their original appointments when CFC is initiated, and they will contribute available slots when they are rescheduled. CFC is a process that is initiated manually or automatically according to a schedule or newly available information, such as, time slots become available and it is needed to reschedule. It ends when the iteration of rescheduling is done. The system process is described as follows, where $\Omega^{\prime \prime}\left(\subseteq \Omega^{\prime}\right)$ is a new set of available time slots after one iteration of rescheduling, $\Omega^{\prime}=\Omega^{\prime \prime}$ expresses that no new slot is allocated in the rescheduling process.

CallForCollaboration Process:

Input: $\Omega^{\prime}$

Output: $T^{\prime}$

Repeat

Step 1: Receive: $\Pi, W$ and $P^{\prime}$;

Until (time is due).

Repeat

Step 2: Rescheduling $\left(\Omega^{\prime}, \Pi, W, P^{\prime}, \Omega^{\prime \prime}, T^{\prime}\right)$; Until $\left(\Pi=\Phi \vee \Omega^{\prime}=\Omega^{\prime \prime}\right)$;

Send out or post new schedules $T^{\prime}$;

Rescheduling Process:

Input: $\Omega^{\prime}, \Pi, W, P^{\prime}$ 
Output: $T^{\prime}$ and $\Omega^{\prime \prime}$.

Step 1: Maximize $\Delta$ while their preferences are satisfied;

Step 2: Form and retürn $T^{\prime}$ and $\Omega^{\prime \prime}$.

To decrease the search space in the re-scheduling process, we propose to introduce some restrictions in a bidding process:

1) Each round of CFC considers a group of continuous time slots as a whole, called time slot block or simply block with a size attached (i.e., the number of continuous time slots). Now, we obtain a set of available blocks $\Omega$. A size vector $S: \Omega \rightarrow \mathcal{N}=\{0,1,2, \ldots\}$ is introduced, where $S[i]$ expresses the size of block $i(i \in \Omega)$;

2) Patients may choose some from these blocks (See Table VII, the available blocks have different number of slots) and specify how many slots they require. A size vector, $S^{\prime}: \Pi \rightarrow \mathcal{N}$ is introduced, where $S^{\prime}[j]$ expresses the requested size of the requested block by patient $j(j \in \Pi)$; 3) The sequence of the outpatients' choices shows their preferences; and

4) A patient is allocated at most one block with the requested size.

TABle VII. The AVAILABle Time Blocks (10) FOR TABLE I.

\begin{tabular}{|c|c|c|c|c|c|c|c|c|c|c|}
\hline & Mon & Tue & Wed & Thu & Fri & Mon & Tue & Wed & Thu & Fri \\
\hline $8: 00$ & \multirow[t]{2}{*}{0} & & & & & & & & & \\
\hline 9:00 & & & \multirow[t]{3}{*}{3} & & & & & \multirow[t]{3}{*}{6} & & \\
\hline $10: 00$ & & \multirow[t]{3}{*}{2} & & & & & & & & \\
\hline 11:00 & & & & & & & & & \multirow[t]{2}{*}{8} & \\
\hline 12:00 & & & & & & & & & & \\
\hline 13:00 & & & & & & & & \multirow[t]{4}{*}{7} & & \\
\hline 14:00 & & & & \multirow[t]{3}{*}{4} & & & \multirow[t]{4}{*}{5} & & \multirow[t]{6}{*}{9} & \\
\hline $15: 00$ & \multirow[t]{4}{*}{1} & & & & & & & & & \\
\hline $16: 00$ & & & & & & & & & & \\
\hline $17: 00$ & & & & & & & & & & \\
\hline $18: 00$ & & & & & & & & & & \\
\hline 19:00 & & & & & & & & & & \\
\hline
\end{tabular}

As for the problem in Table I, we may redraw it shown as in Table VII. Now, the problem can be re-specified. We introduce a function $g: \Omega^{*} \times \Pi \rightarrow[0,1]$, where $\Omega^{*}$ is the power set of $\Omega$, as a preference scale to evaluate the relative preference among different patients and requested bundles, i.e., $g(i, j)=$

$$
\begin{cases}\left(k_{j}-l\right) / k_{j} & l=0,1, \ldots, k_{j}-1, i \in P_{j}, i=P_{j}[l] ; \\ 0 & \text { otherwise. }\end{cases}
$$

$h(i, j)$ is introduced to evaluate the matching scales of the request, i.e.,

$$
h(i, j)=\left\{\begin{array}{ll}
S^{\prime}[j] / S[i] & \left(S^{\prime}[j] \leq S[i]\right) \\
0 & \left(S^{\prime}[j]>S[i]\right)
\end{array}(0 \leq i<\mathrm{m}, 0 \leq j<\mathrm{n}) \quad \ldots(7)\right.
$$

For example, suppose that other conditions are the same. If patient $x$ bids for the $3^{\text {rd }}(i=2)$ choice in 5 choices $\left(k_{x}=5\right)$; and patient $y$ bids for the $2^{\text {nd }}(i=1)$ in 3 choices $\left(k_{y}=3\right)$, we prefer the latter, i.e., $y$, because $g(2, x)=3 / 5<g(2, y)=2 / 3$. If patient $x$ requests 3 slots in a 5 -slot block $z$, i.e., $S[z]=5, S^{\prime}[x]=3$, and patient $y$ requests 4 slots in the same block $z, S^{\prime}[y]=4$, we prefer $y$, because $h(z, x)=S^{\prime}[x] / S[z]=3 / 5<h(z, y)=$ $S^{\prime}[y] / S[z]=4 / 5$.

With this adjustment, the outpatient scheduling problem becomes to find an assignment scheme for all patients such that $\Delta_{1}$ is maximized; all patient preferences are best satisfied; and all patient requests are best matched.

It can be re-formalized as a three-objective optimization problem:

$$
\begin{aligned}
& \max \Delta_{l=} \sum_{i \in \Omega} \sum_{j \in \Pi} x_{j}(i) \times S^{\prime}[j] \times W_{j} \\
& \max \sum_{i \in \Omega} \sum_{j \in \Pi} x_{j}(i) \times g(i, j) \\
& \max \sum_{i \in \Omega} \sum_{j \in \Pi} x_{j}(i) \times h(i, j)
\end{aligned}
$$

subject to

$$
\begin{aligned}
& \forall j \in \Pi, \sum_{i \in \Omega} x_{j}(i) \leq 1 \\
& \forall j \in \Pi, \sum_{i \in \Omega} x_{j}(i)=\sum_{i \in P_{j}} x_{j}(i) \\
& \forall i \in \Omega, \sum_{j \in \Pi} x_{j}(i) \times S^{\prime}[j] \leq S[i] \\
& \forall i \in \Omega, j \in \Pi, x_{j}(i) \in\{0,1\}
\end{aligned}
$$

where (11) ensures that any outpatient can only obtain one from the available time blocks; (12) ensures that if a timeslot block is assigned to an outpatient, it must belong to the block set the outpatient has requested; constraint (13) tells that each block can be allocated to more than one patient, however, the total requested sizes of the assigned patients should not be larger than the size of the original block; and (14) is a $0-1$ constraint. It is evident that the bundle requirement is removed and therefore the problem is simplified.

Note that this is a typical multi-objective optimization problem to which a simple solution is weighted sum [16]. However, it is still time-consuming based on the model (6)(14). For example, a random case $(\mathrm{m}, \mathrm{n}=14)$ cost 54 minutes to be solved by a weighted sum method by using ILOG (The Model and Data I in Section VI of the multimedia document). TABLE VIII. AN EXAMPLE FOR OUTPATIENT SCHEDULING

\begin{tabular}{|l|l|l|l|l|l|}
\hline ID & Name & $\begin{array}{l}\text { Priority } \\
\text { value }\end{array}$ & $\begin{array}{l}\text { Patients' } \\
\text { biddings } \\
\text { for } \\
\text { blocks }\end{array}$ & $\begin{array}{l}\text { Block } \\
\text { size }\end{array}$ & Original appointment \\
\hline P1 & Tom & 3 & $\begin{array}{l}1,3,6, \\
8\end{array}$ & 2 & $\begin{array}{l}14(8-9 \text { of Thursday in } \\
\left.\text { the } 3^{\text {rd }} \text { week }\right)\end{array}$ \\
\hline P2 & Chris & 2 & 1,5 & 3 & $\begin{array}{l}12(12-14 \text { of Monday } \\
\left.\text { in the } 4^{\text {th }} \text { week }\right)\end{array}$ \\
\hline P3 & Ana & 3 & $3,6,8$ & 1 & $\begin{array}{l}10(10 \text { of Thursday in } \\
\left.\text { the } 3^{\text {rd }} \text { week }\right)\end{array}$ \\
\hline P4 & Bob & 2 & $3,6,8$ & 2 & $\begin{array}{l}13(10-11 \text { of Tuesday } \\
\left.\text { in the } 4^{\text {th }} \text { week }\right)\end{array}$ \\
\hline P5 & Don & 1 & $0, . ., 9$ & 1 & $\begin{array}{l}11(17 \text { of Friday in the } \\
\left.3^{\text {rd }} \text { week }\right)\end{array}$ \\
\hline P6 & Jane & 3 & 4,9 & 2 & $\begin{array}{l}15(13-14 \text { of Friday in } \\
\left.\text { the } 4^{\text {th }} \text { week }\right)\end{array}$ \\
\hline
\end{tabular}

Table VIII is the assumed information collected by one round of CFC similar to the requests from Table V. It is assumed that the responses to CFC are for the available blocks from 0 to 9 . The blocks originally assigned to the 6 responded patients are $10-15$. Please note that it is not hard to collect the information shown in Table VIII. For example, a group email can be sent out to all the outpatients. The interested ones can click on a link provided by the e-mail to provide their preferences among the available blocks. The priority values are found based on the electronic documents of outpatients in the health care office. So are the original appointments.

To solve the problem expressed by formula (6-14) efficiently, the GRA algorithm must be iteratively called because it can only assign one available block to each patient in each iteration, i.e., it can only solve the problem by replacing (13) with (15): 


$$
\forall i \in \Omega, \sum_{j \in \Pi} x_{j}(i) \leq 1
$$

After each GRA assignment, some available blocks may be still available for those outpatients who have not yet scheduled. We need more GRA processes until no available time slot blocks cover the requests of outpatients. This iteration may affect the global optimization as described by formula 6-14, but leads to an efficient solution. That the three optimization goals are synthesized to form one goal is another factor to affect the optimal solution. Note that, the major idea is to transfer some constraints to numbers in order to apply the optimization algorithm. We admit that not all the constraints can be transferred to proper numbers, but there are indeed some constraints that can be processed this way.

\section{GROUP ROLE ASSIGNMENT}

By E-CARGO [23-27], we mean Environments, Classes, Agents, Roles, Groups and Objects. To deal with the role assignment problems, we emphasize a role set denoted by $\mathcal{A}$ and an agent set denoted by $\mathcal{R}$. Agents in $\mathcal{A}$ are numbered as 0 , $1, \ldots$, and $m-1(\mathrm{~m}=|\mathcal{A}|)$; and roles in $\mathcal{R}$ are numbered as 0,1 , $\ldots$, and $n-1(\mathrm{n}=|\mathbb{R}|)$.

Definition 1: A role range vector is a vector of the lower ranges of roles denoted as $L[j] \in \mathcal{N}(0 \leq j<n)$.

Definition 2: A qualification matrix is defined as $Q: \mathcal{A} \times \mathbb{R} \rightarrow$ $[0,1]$, where, $Q[i, j]$ expresses the qualification value of agent $i$ for role $j$.

The improvement of the efficiency of the algorithm for the outpatient scheduling problem mainly comes from the formation of the $Q$ matrix.

Definition 3: A role assignment matrix is defined as $T: \mathcal{A} \times \mathbb{R}$ $\rightarrow\{0,1\}$. If $T[i, j]=1$, agent $i$ is assigned to role $j$ and $T[i$, $j]=0$ otherwise $(0 \leq i<m ; 0 \leq j<n)$. Note that $\mathrm{T}$ also expresses a group.

Definition 4: A group qualification is defined as the sum of the assigned agents' qualifications, i.e., $\sum_{i=0}^{m-1} \sum_{j=0}^{n-1} Q[i, j] \times T[i, j]$.

Definition 5: A role $r$ is workable if it is assigned enough current agents to play it, i.e., $\sum_{i=0}^{m-1} T[i, j] \geq L[j]$ [23-27].

Definition 6: A group expressed by $T$ is workable if all its roles are workable.

Definition 7: The group role assignment (GRA) problem. is to find an assignment matrix $\mathrm{T}$ that makes the group qualification is the largest, i.e., $\max \left\{\sum_{i=0}^{m-1} \sum_{j=0}^{n-1} Q[i, j] \times T[i, j]\right\}$ subject to $\forall j\left(\sum_{i=0}^{m-1} T[i, j]=L[j]\right)(0 \leq j<n)$.

\section{From the OutPatient PRoblem to the Group Role ASSIGNMENT PROBLEM}

In terms of GRA, we consider patients as roles and time slot blocks as agents. This consideration is explained next.

Definition 8: An original agent vector $V$ is an $n$-vector of the original agent assigned for a role, i.e., $V: R \rightarrow \mathcal{A}$, where $V[j]$ means the agent originally assigned to role $j$.

We use $F^{t}(P, i)$ to express the set formed by the elements of vector $P[i]$. If $F^{t}(P, j) \cap F^{t}\left(P, j^{\prime}\right) \neq \Phi$, we say that roles $j$ and $j^{\prime}$ are competing on agents (blocks) expressed by $F^{t}(P, j) \cap F^{t}(P$, $j^{\prime}$ ). We keep other symbols as described in Sections III and IV.

In solving the outpatient scheduling problem, the most important step is to build an appropriate $Q$ to reflect the values required in the assignment process. The vectors $W$ and $P$ and functions $g$ and $h$ can be used to form $Q$ with the following formula $(0 \leq i<m, 0 \leq j<n)$ :

$$
Q[i, j]=\zeta(P, i, j) \times g(i, j) \times h(i, j) \times \gamma(i) \times\left(W[j] / w_{\max }\right) ;
$$

where,

$$
\begin{aligned}
& \zeta(P, i, j)= \begin{cases}1 & i \in F^{t}(P, j) ; \\
0 & i \notin F^{t}(P, j) ;\end{cases} \\
& \gamma(j)=S^{\prime}[j] / \max \left\{\mathrm{S}^{\prime}[0], \mathrm{S}^{\prime}[1], \ldots, \mathrm{S}^{\prime}[n-1]\right\}
\end{aligned}
$$

Note that, $\zeta(P, i, j)$ tells that time slot blocks (agents) are only qualified for the requested roles (patients), i.e., it is to prevent the situation that an agent (time slot block) is assigned a role (patient) who is not willing to accept; $\gamma(j)$ considers the size of the assigned blocks to patient $j$ and note that we do not use $S^{\prime}[j]$ directly in order to keep $Q[i, j] \in[0,1] ; g(i, j)$ in (6) ensures that if two available blocks are patient $j$ 's choices, the block with a better preference is assigned, and that if two patients are competing for one time block, the patients' preferences are serialized, e.g., if time block $z$ is patient $x$ 's $2^{\text {nd }}$ preference (its index is 1 ) in 3 choices and is patient $y^{\prime} s 2^{\text {nd }}$ preference (its index is 1$)$ in 5 choices then y is preferred $(2 / 3$ $<4 / 5)$; and $h(i, j)$ in (7) expresses that a time block (agent) has higher qualification if its size fits the time block size better.

$\left[\begin{array}{llllll}0.000 & 0.000 & 0.000 & 0.000 & 0.056 & 0.000 \\ 0.333 & 0.500 & 0.000 & 0.000 & 0.025 & 0.000 \\ 0.000 & 0.000 & 0.000 & 0.000 & 0.030 & 0.000 \\ 0.333 & 0.000 & 0.111 & 0.296 & 0.026 & 0.000 \\ 0.000 & 0.000 & 0.000 & 0.000 & 0.022 & 0.444 \\ 0.000 & 0.250 & 0.000 & 0.000 & 0.014 & 0.000 \\ 0.167 & 0.000 & 0.056 & 0.148 & 0.011 & 0.000 \\ 0.000 & 0.000 & 0.000 & 0.000 & 0.008 & 0.000 \\ 0.167 & 0.000 & 0.056 & 0.148 & 0.011 & 0.000 \\ 0.000 & 0.000 & 0.000 & 0.000 & 0.002 & 0.111\end{array}\right]$

Fig. 1. The $Q$ matrix for Table VIII.

From Table VIII, $W=[3,2,3,2,1,3], w_{\max }=3, P=[[1,3$, $6,8],[1,5],[3,6,8],[3,6,8],[0,1,2,3,4,5,6,7,8,9]$, [4, 9]], $S^{\prime}=[2,3,1,2,1,2], S=[2,4,3,3,3,4,4,4,2,6]$, and $V=$ $[10,11,12,13,14,15]$. For example, $Q[3,2]=1 \times(1 / 3) \times(1 / 3)$ $\times[(3-0) / 3] \times(3 / 3)=0.111$.

By synthesizing all the above data, we get a qualification matrix $Q$ shown in Fig. 1.

Now, $\mathcal{A}$ is the set of $m$ blocks available at the time of CFC; $R$ is the set of $n$ patients who have responded to CFC; and $Q$ is an $m \times n$ matrix obtained by formula (16-18). Then, the outpatient scheduling problem is in fact becoming a GRA problem to find an $m \times n$ assignment matrix $\mathrm{T}$ to 


$$
\begin{aligned}
& \max \left\{\sum_{i=0}^{m-1} \sum_{j=0}^{n-1} Q[i, j] \times T[i, j]\right\} \\
& \text { subject to } \\
& \sum_{j=0}^{n-1} T[i, j] \leq 1(0 \leq i<m) \\
& \forall j\left(\sum_{i=0}^{m-1} T[i, j]=\mathrm{L}[j]\right)(0 \leq j<n)
\end{aligned}
$$

In E-CARGO, that one agent can only play one role in GRA directly follows the above requirement of outpatient scheduling that one block (agent) can be assigned to only one outpatient (role), i.e., $\sum_{j=0}^{n-1} T[i, j] \leq 1(0 \leq i<m)$.

In fact, we can even loosen the restriction in GRA, i.e., remove the requirement of $\forall j, \sum_{i=0}^{m-1} T[i, j]=\mathrm{L}[j](0 \leq j<n)$,

because it is acceptable for some bidding outpatients to obtain no block in one round of CFC.

Based on the GRA algorithm, we can obtain matrix $T$ shown as in Fig. 2 for the $Q$ matrix in Fig. 1.

$\left[\begin{array}{llllll}0 & 0 & 0 & 0 & 1 & 0 \\ 0 & 1 & 0 & 0 & 0 & 0 \\ 0 & 0 & 0 & 0 & 0 & 0 \\ 1 & 0 & 0 & 0 & 0 & 0 \\ 0 & 0 & 0 & 0 & 0 & 1 \\ 0 & 0 & 0 & 0 & 0 & 0 \\ 0 & 0 & 0 & 1 & 0 & 0 \\ 0 & 0 & 0 & 0 & 0 & 0 \\ 0 & 0 & 1 & 0 & 0 & 0 \\ 0 & 0 & 0 & 0 & 0 & 0\end{array}\right]$

Fig. 2. The Assignment Matrix $T$.

Let $x_{j}(i)$ in $(8)=T[i, j](0 \leq i<m ; 0 \leq j<n)$. We obtain $\Delta_{1}{ }^{*}$ as 26. We can translate the matrix $T$ in Fig. 2 to a list of assignment tuples as the scheduling result of the $1^{\text {st }}$ round CFC (named slots in Table VIII).

TABle IX. The AsSigned Time SLOTS.

\begin{tabular}{|l|l|l|l|l|l|l|l|l|l|l|}
\hline & Mon & Tue & Wed & Thu & Fri & Mon & Tue & Wed & Thu & Fri \\
\hline $8: 00$ & Don & & & & & & & & & \\
\hline $9: 00$ & & & Tom & & & & & Bob & & \\
\hline $10: 00$ & & & Tom & & & & & Bob & & \\
\hline $11: 00$ & & & & & & & & & Ann & \\
\hline $12: 00$ & & & & & & & & & & \\
\hline $13: 00$ & & & & & & & & & & \\
\hline $14: 00$ & & & & Jane & & & & & & \\
\hline $15: 00$ & Chris & & & Jane & & & & & & \\
\hline $16: 00$ & Chris & & & & & & & & & \\
\hline $17: 00$ & Chris & & & & & & & & & \\
\hline $18: 00$ & & & & & & & & & & \\
\hline $19: 00$ & & & & & & & & & & \\
\hline
\end{tabular}

TABle X. The New Available Time Slots.

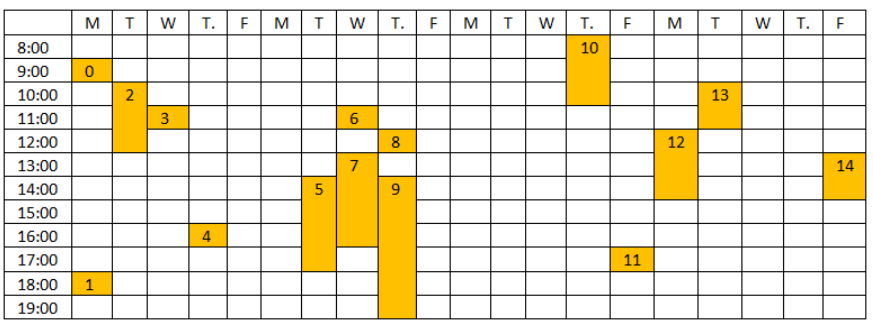

Combined with the original agent (time slot block) vector $V$, the new list of available time slot blocks becomes (shaded blocks Table X). Because all the patients are scheduled, no more iteration of GRA is required. The above list can be used for the $2^{\text {nd }}$ round of CFC. Note that block 10 is formed by combining two original appointments, i.e., (slots 8-9 and slot 10 of Thursday in the $3^{\text {rd }}$ week).

\section{THE ALGORITHM AND COMPLEXITY}

The complexity of the efficient GRA algorithm is polynomial [27]. If we transfer the outpatient scheduling problem into the GRA problem, the outpatient scheduling problem is solved in polynomial time. The following algorithm OutpatientRescheduling mainly describes the pre-process, the use of the GRA algorithm, and the post-process.

Note: the following algorithm is described in a Java-like language; " $a=b$ " means to check if $a$ is equivalent to $b$ and " $a:=b$ " is to assign the value of $b$ to $a$.

\section{Input:}

$\Pi$ : A set of outpatients' bidding $<\mathrm{x}, \mathrm{y}, \mathrm{Z}, \mathrm{v}>$, where $\mathrm{x}$ is the identification of the outpatient; $y$ : the priority value of the outpatient; $\mathrm{Z}$ is the list of the bids of time slot blocks of the outpatient, where the position of a block in the list expresses the patient's preference; $u$ is the size of the requested block; and $\mathrm{v}$ is the original time slot block of the outpatient.

$\Omega$ : A set of the available time slot blocks expressed as $<\mathrm{c}^{\prime}$, $d^{\prime}, e^{\prime}, f^{\prime}>$, where $c^{\prime}$ is the starting slot number; $d^{\prime}$ is size of the block; $\mathrm{e}^{\prime}$ is the day number of the week; and $\mathrm{f}^{\prime}$ is the number of the week.

Output:

$\chi$ : A list of tuples $\langle a, b\rangle$ where $a$ means a patient and $b$ means a time slot block.

$\Omega$ : a new set of available time slot blocks.

\section{OutpatientRescheduling $(\Pi, \Omega, \chi)$}

$\left\{m_{l}:=m:=|\Omega|\right.$;

$n_{l}:=n:=|\Pi|$

while $\left(m_{1}>0\right.$ and $\left.n_{l}<n\right)$;

\section{\{ Step 1:}

Transfer $\Omega$ into a list $\Omega$, where ' $\Omega[\mathrm{i}] \in \Omega ;(0 \leq i \leq m-1)$;

\section{Step 2:}

Transfer $\Pi$ into 4 lists $\mathrm{P}, \mathrm{W}, \mathrm{C}$, and $\mathrm{V}$, i.e., $\mathrm{P}[j]:=\mathrm{c} . \mathrm{x}$, $\mathrm{W}[j]:=c . y, C[j]:=c . Z, V[j]:=c . v(c \in \Pi, 0 \leq j \leq n-1)$;

Step 3: Note that $\mathrm{h}, \mathrm{g}, \mathrm{Q}, \zeta$, and $\gamma$ are all $m \times n$ matrices corresponding to formula $(6,7,16-18)$.

$$
\begin{aligned}
& \mathrm{W}_{\max }:=\max \{\mathrm{c} . \mathrm{y} \mid(\mathrm{c} \in \Pi)\} \\
& \text { for }(0 \leq i \leq m-1,0 \leq j \leq n-1)\{ \\
& \quad \text { if }(i \in C[j]) \zeta[i, j]:=1 ; \\
& \quad \text { else } \zeta[i, j]:=0 ; \\
& \quad \text { if }\left(S^{\prime}[j] \leq S[i]\right) \mathrm{h}[i, j]:=S^{\prime}[j] / S[i] ; \\
& \quad \text { else } \mathrm{h}[i, j]:=0 ; \\
& \alpha[j]:=C[j] . \text { length; } \\
& \quad \beta[i, j]:=\text { index of } i \text { in } C[j] ; \\
& \quad \mathrm{g}[i, j]:=(\alpha(C, j)-\beta(C, i, j)) / \alpha(C, j) ; \\
& \quad \mathrm{Q}[i, j]:=\zeta[i, j] \times \mathrm{h}[i, j] \times \mathrm{g}[i, j] \times \gamma[j] \times \mathrm{W}[j] / \mathrm{w}_{\max } ; \\
& \}^{\prime}
\end{aligned}
$$

Step 4: Note $\mathrm{T}$ is an $m \times n$ matrix.

Initialize the assignment matrix $\mathrm{T}$ with $\{0\}$;

Call RatedAssignForOutpatients $(\mathrm{Q}, \mathrm{T}, m, n)$;

Step 5: Form the new list of appointments in <patient, time slot block> and adjust the available time slot blocks.

Initialize $\chi[j]$ with NULL $(0 \leq j \leq n-1)$;

for $(0 \leq i \leq m-1,0 \leq j \leq n-1)$

if $(\mathrm{T}[i, j]=1)\{$ 


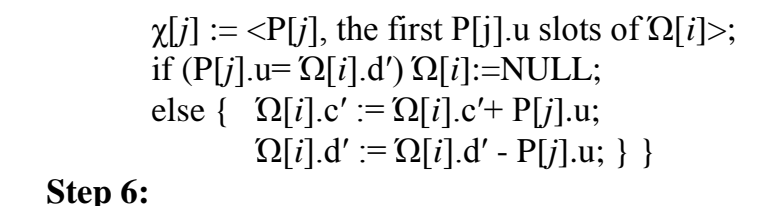

Step 6:

Keep the unscheduled patients the original appointments.

$\Pi:=\Phi$;

for $(0 \leq j \leq n-1)$

if $(\chi[j]=$ NULL $)\{$

$\chi[j]:=<\mathrm{P}[j], \mathrm{V}[j]>$;

$\mathrm{V}[j]:=\mathrm{NULL} ;$

$\Pi:=\Pi \cup\{\mathrm{P}[j]\} ;\}$

\section{Step 7:}

Form the new set of available time slot blocks.

$\Omega:=\Phi$;

for $(0 \leq i \leq m-1)$

if $(\Omega[i] \neq$ NULL $) \Omega:=\Omega \cup\{\Omega[i]\}$;

for $(0 \leq j \leq n-1)$

if $(\mathrm{V}[j] \neq \mathrm{NULL}) \Omega:=\Omega \cup\{\mathrm{V}[j]\}$;

$m_{1}:=|\Omega|$;

$n_{1}:=|\Pi| ;$

\}

if $\left(n_{l}=0\right)$ every patient gets an assignment;

else some patients have no assignments;

\}//End of OutpatientRescheduling

The algorithm RatedAssignForOutpatients is described as follows.

Input: Q: an $m \times n$ rated qualification matrix.

Output: T: an $m \times n$ assignment matrix.

RatedAssignForOutpatients $(\mathrm{Q}, \mathrm{T}, m, n)$

$\{$ Step 1: $k=\max \{m, n\}$;

Transfer the $m \times n$ matrix $\mathrm{Q}$ to a $k \times k$ matrix $\mathrm{M}$ [29];

Step 2: K-M (M); //Call the K-M algorithm;

Step 3: Form the assignment matrix $\mathrm{T}$ based on the result of

K-M (M);

Step 4: return T;

\}

Note that, in the above algorithm, the requirement of $\forall j, \sum_{i=0}^{m-1} T[i, j]=\mathrm{L}[j](0 \leq j<n)$ in the RatedAssign algorithm is

removed, because it is acceptable for some bidding outpatients to obtain no block in one round of CFC.

Theorem 1: Algorithm RatedAssignForOutpatients has the complexity of $O\left(k^{3}\right)(k=\max \{m, n\})$.

Proof:

Step 1 has the complexity of $O\left(k^{2}\right)$.

Step 2 (K-M algorithm) has $O\left(k^{3}\right)[11,14]$

Step 3 has $O\left(k^{2}\right)$.

Step 4 has $O(1)$.

Therefore, the total complexity is $O\left(k^{3}\right)$.

Theorem 2: Algorithm OutpatientRescheduling has the complexity between $O\left(k^{3}\right)$ (the best case) and $O\left(\delta k^{3}\right)$ (the worst case), where $k=\max \{m, n\}$ and $\delta=\min \{m, n\}$.

Proof:

Based on Theorem 1, Step 4 has the complexity of $O\left(k^{3}\right)$.

Step 1 has $O(m)$.

Step 2 has $O(n)$.

Step 3 has $O(m \times n)$.
Step 5 has $O(m \times n)$.

Step 6 has $O(n)$.

Step 7 has $O(m+n)$.

Therefore, The total complexity inside the do loop is $O\left(k^{3}\right)$.

As for the while loop, the complexity is mainly determined by three factors besides $m$ and $n$ :

(1) the original appointments brought in by the bidding outpatients;

(2) the conflict bidding for the same blocks; and

(3) the requested blocks from patients.

The worst situation is that the number of the do loops is $\delta=$ $\min \{m, n\}$, i.e., all the patients are bidding for the same block that is large enough for all the patients, and each do loop satisfies one patient within this block.

The best is within one loop:

(1) All the patients bid for different blocks and are satisfied in one loop; or

(2) All the blocks are assigned to patients in one loop.

In summary, the complexity of algorithm OutpatientRescheduling is between $O\left(k^{3}\right)$ and $O\left(\delta k^{3}\right)$.

\section{VERIFICATIONS AND COMPARISONS}

To verify the proposed approach, we conducted simulations, performance experimentations, and performance comparisons. The simulations show that the optimality is satisfied. The times used by the proposed algorithm for some random problems are from 0.44 milliseconds (ms) to $2 \mathrm{~ms}$ compared with that of ILOG from $280 \mathrm{~ms}$ to more than 20 minutes. The performance experiments show that the time used for large groups $(\mathrm{m}=80$, $\mathrm{n}=1000$ ) is practical, i.e., at most 6.6 seconds and in average 4.16 seconds. All the results are included in the supplemental multimedia document.

\section{CONClusions And Future Work}

This paper contributes an efficient approach to outpatient scheduling by a special treatment for collecting patients' choices from available time slots. An exciting future task will be to generalize the proposed approach and to find a way to transform as many constraints as possible into a qualification matrix of GRA. If all the constraints of a general scheduling problem can be transformed into a qualification matrix of GRA in polynomial time, such a general scheduling problem will be solved within polynomial time. Such idea may be extended to other scheduling problems [1, 3, 12, 15, 19].

More interest future tasks include: 1) to implement an online service system in real health care environments; 2 ) to find out an algorithm if functions $h$ and $g$ are correlated; 3) to investigate if the abnormality in Figs. 3 and 4 of the supplement document is a determined phenomenon; 4) to introduce heuristics in solving such assignment problems; and 5) to conduct empirical studies on the proposed approach of Call for Collaboration.

\section{REFERENCES}

[1] B. Alidaee, H. Wang, and F. Landram, "On the Flexible Demand Assignment Problems: Case of Unmanned Aerial Vehicles", IEEE Trans. on Automation Sci. and Eng., 8(4), pp. 865-868, Oct. 2011. 
[2] T. Cayirli, and E. Veral, "Outpatient scheduling in health care: a review of literature", Production and Operations Management, Jan. 1 2003, avail: http://www.allbusiness.com/health-care/health-care-facilitiesclinics/10619562-1.html .

[3] C. F. Chu, M. Zhou, H. Chen, and Q. Shen, "A Polynomial Dynamic Programming Algorithm for Crude Oil Transportation Planning," IEEE Trans. on Automation Sci. and Eng., 9(1), pp. 42-55, Jan. 2012.

[4] P. Cramton, Y. Shoham, and R. Steinberg. "Introduction to Combinatorial Auctions", P. Cramton, et al. (Ed.), Combinatorial Auctions, Cambridge, MA: MIT Press, pp. 1-13, 2006.

[5] P. Godin, C. Wang, "Agent-Based Outpatient Scheduling for Diagnostic Services", Proc. of The IEEE Int'l Conf. on Systems, Man and Cybernetics (ICSMC), Istanbul, Turkey, pp. 1851-1856, 2010.

[6] S. Gul, B. T. Denton, J. W. Fowler, and T. Huschka, "Bi-Criteria Scheduling of Surgical Services for an Outpatient Procedure Center," Production and Operations Management, vol. 20, no. 3, May/June 2011, pp. 406-417.

[7] M. Guo, M. Wagner, and C. West, "Outpatient Clinic Scheduling - A Simulation Approach", in Proc. of the $36^{\text {th }}$ Winter Simulation Conference, Washington, DC, USA, pp. 1981-1987, 2004.

[8] D. Gupta, and B. Denton, "Appointment scheduling in health care: Challenges and opportunities", IIE Trans., vol. 40, 800-819, 2008.

[9] IBM, ILOG CPLEX Optimizer, avail: http://www-01.ibm.com/ software/integration/optimization/cplex-optimizer/, April, 2011.

[10] G. C. Kaandorp, and G. Koole, "Optimal outpatient appointment scheduling", Health Care Manag. Sci., vol. 10, no. 3, pp. 217-229, 2007.

[11] H. W. Kuhn, "The Hungarian method for the assignment problem", Naval Research Logistic Quarterly, vol. 2, 1955, pp. 83-97.

[12] H. C. Lau, Z. J. Zhao, S. S. Ge, and T. H. Lee, "Allocating Resources in Multiagent Flowshops with Adaptive Auctions", IEEE Trans. on Automation Sci. and Eng., vol. 8, no. 4, pp. 732-743, Oct. 2011.

[13] N. Liu, S. Ziya, and V. G. Kulkarni, "Dynamic Scheduling of Outpatient Appointments Under Patient No-Shows and Cancellations", Manufacturing \& Service Operations Management, vol. 12, no. 2, Spring 2010, pp. 347-366.

[14] J. Munkres, "Algorithms for the assignment and transportation problems", Journal of the Society for Industrial and Applied Mathematics, vol. 5, no. 1, March 1957, pp. 32-38.

[15] V. Ng and B. Chan, "Quality Assignments for WSDL-Based Services", Proc. of Computer Supported Cooperative Work in Design II, Lecture Notes in Computer Science, 2006, vol. 3865, pp.163-173.

[16] J. Patrick and M. L. Puterman, "Improving resource utilization for diagnostic services through flexible inpatient scheduling: A method for improving resource utilization," Journal of the Operational Research Society, vol. 58, pp. 235-245, 2006.

[17] A. Rais and A. Viana, "Operations Research in Healthcare: a survey", Int'l Trans. in Operational Research, vol. 18, pp. 1-31, 2010.

[18] R. L. Rardin, Optimization in Operations Research, Prentice Hall, Upper Saddle River, New Jersey, 1998.

[19] P. Santibáñez, M. Begen, D. Atkins, "Surgical block scheduling in a system of hospitals: an application to resource and wait list management in a BC health authority," Health Care Management Science, vol. 10, pp. 269-282, 2007

[20] I. B. Vermeulen, S. M. Bohte, S. G. Elkhuizen, J.S. Lameris, P.J.M. Bakker, and J.A. La Poutre, "Adaptive Resource Allocation for Efficient Patient Scheduling", Artificial Intelligence in Medicine, vol. 46, no. 1, pp. 67-80, May 2009.

[21] N. Wu, and M. C. Zhou, "Schedulability Analysis and Optimal Scheduling of Dual-Arm Cluster Tools with Residency Time Constraint and Activity Time Variation," IEEE Trans. on Automation Science and Engineering, vol. 9, no. 1, pp. 203-209, Jan. 2012.

[22] M. B. Wright, "Speeding up the Hungarian algorithm", Computers \& Operations Research, vol. 17, no. 1, pp. 95-96, 1990.

[23] H. Zhu and M.C. Zhou, "Role-Based Collaboration and its Kernel Mechanisms", IEEE Trans. on SMC, Part C, vol. 36, no. 4, pp. 578589 , July 2006

[24] H. Zhu and M.C. Zhou, "Role Transfer Problems and Algorithms", IEEE Trans. on SMC, Part A, vol. 36, no. 6, pp. 1442-1450, Nov. 2008.

[25] H. Zhu and M.C. Zhou, "M-M Role Transfer Problems and Solutions", IEEE Trans. on SMC, Part A, vol. 39, no. 2, pp. 448-459, March 2009.
[26] H. Zhu, and M. Zhou, "An Efficeint Solution to the Role Transfer Problem," IEEE Trans. on SMC, Part A, vol.42, no.2, pp. 491-496, March 2012.

[27] H. Zhu, M. Zhou, and R. Alkins, "Group Role Assignment via a KuhnMunkres Algorithm-based Solution," IEEE Trans. on SMC, Part A, vol.42, no. 3, 2012, pp. 739-750.

Haibin Zhu (M'02-SM'04) is Full Professor of the Department of Computer Science and Mathematics, Director and Founder of Collaborative Systems Laboratory, Nipissing University, Canada. He has published 120+ research papers, four books and two book chapters. He is serving and served as cochair of the Technical Committee (TC) of Distributed Intelligent Systems of IEEE SMC Society, guest (co-) editor for 3 special issues of prestigious journals, and organization chairs for many IEEE conferences. $\mathrm{He}$ is a founding researcher of Role-Based Collaboration and Adaptive Collaboration. $\mathrm{He}$ is the receipt of many awards. His research interests include Collaboration Theory, Technologies, Systems, and Applications, Human-Machine Systems, Multi-Agent Systems, and Distributed Intelligent Systems. For more information please feel free to browse Dr. Zhu's Website at http://faculty.nipissingu.ca/haibinz/.

Ming Hou (M'05-SM'07) received the Ph.D. degree in human factors engineering from the University of Toronto, Toronto, ON, Canada, in 2002.

He is currently a Defence Scientist and the Head of the Advanced Interface Group, Defence Research and Development Canada-Toronto, where he is responsible for providing informed decisions to the Canadian Forces on investment in and application of advanced technologies for operator machine interface requirements. His research interests include applied cognition, intelligent adaptive system design, virtual/mixed reality, supervisory control of uninhabited vehicles, and e-learning. Dr. Hou is a member of the Human Factors and Ergonomics Society and the Association of Computing Machinery. He was the Chair of the Symposium on Human Factors and Ergonomics at the 2009 IEEE Toronto International Conference Science and Technology for Humanity. He has been the Co-Chair of the International Symposium on Mixed and Virtual Reality since 2004.

Chun Wang (M'06) received the B.Eng. degree in 1990 from Huazhong University of Science and Technology, China, and the M.E.Sc. and Ph.D. degrees in computer engineering in 2004 and 2008, respectively, from the University of Western Ontario, Canada. He is currently an assistant professor at Concordia Institute for Information Systems Engineering, Concordia University, Montreal, Quebec, Canada. He worked as a software engineer and a project manager for The China National Petroleum Co. from 1990 to 2000. His research focuses on e-Supply Chain, e-Commerce, algorithmic mechanism design, and multi-agent systems.

MengChu Zhou (S'88-M'90-SM'93-F'03) received his B.S. degree in Electrical Engineering from Nanjing Univ. of Sci. and Tech., Nanjing, China in 1983, M.S. degree in Automatic Control from Beijing Inst. of Tech., Beijing, China in 1986, and $\mathrm{Ph}$. D. degree in Computer and Systems Engineering from Rensselaer Polytechnic Inst., Troy, NY in 1990. He joined New Jersey Inst. of Tech. (NJIT), Newark, NJ in 1990, and is currently a Professor of Electrical and Computer Engineering and the Director of Discrete-Event Systems Laboratory. He is presently a Professor at Tongji University, Shanghai, China. His research interests are in intelligent automation, lifecycle engineering and sustainability evaluation, Petri nets, wireless ad hoc and sensor networks, semiconductor manufacturing, Web service, workflow, and energy systems. He has over 440 publications. He was invited to lecture in many countries and served as a plenary speaker for many int'l conferences. He is a founding Editor of IEEE Press Book Series. He served as Associate Editor of IEEE Trans. on Robotics and Automation (1997-2000), and IEEE Trans. on Automation Sci. and Eng.(2004-2007), and is currently Editor of IEEE Trans. on Automation Sci. and Eng., and Associate Editor of IEEE Trans. on SMC-Part A and IEEE Trans. on Industrial Informatics. He served as Guest-Editor for many prestigious journals including IEEE Trans. on Industrial Electronics. He was General Chairs for many int'l conferences. Dr. Zhou has led or participated in projects with total budget over $\$ 10 \mathrm{M}$. He was the recipient of many prestigious awards. He was recently elevated to Fellow of American Association for the Advancement of Science (AAAS). For more information please feel free to browse Dr. Zhou's Website at http://web.njit.edu/ zhou/. 